This is the submitted version of the article:

González-Guerrero A.B., Maldonado J., Herranz S., Lechuga L.M.. Trends in photonic lab-on-chip interferometric biosensors for point-of-care diagnostics. Analytical Methods, (2016). 8. : 8380 - . 10.1039/c6ay02972h.

Available at: https://dx.doi.org/10.1039/c6ay02972h 


\section{Analytical Methods}

\section{Trends in photonic lab-on-chip interferometric biosensors for point-of-care diagnostics}

\begin{tabular}{|r|l|}
\hline Journal: & Analytical Methods \\
\hline Manuscript ID & Draft \\
\hline Article Type: & Minireview \\
\hline Date Submitted by the Author: & n/a \\
\hline Complete List of Authors: & $\begin{array}{l}\text { Gonzalez-Guerrero, Ana Belén; Catalan Institute of Nanoscience and } \\
\text { Nanotechnology } \\
\text { Maldonado, Jesus; Catalan Institute of Nanoscience and Nanotechnology } \\
\text { Herranz, Sonia; ICN2 } \\
\text { Lechuga, Laura; Institut Catala de Nanotecnologia, }\end{array}$ \\
\hline
\end{tabular}

SCHOLARONE ${ }^{\text {Im }}$

Manuscripts 
Dear Editor,

Please find enclosed for your consideration the manuscript entitled Trends in photonic lab-on-chip interferometric biosensors for point-of-care diagnostics by González-Guerrero et al.

The manuscript is an invited review to the themed collection on portable instrumentation \& point of care technologies for Analytical Methods guest edited by Professors Zheng Ouyang and Jean-Francois Masson.

The work in this manuscript is unpublished and has not been submitted elsewhere for publication.

Sincerely yours,

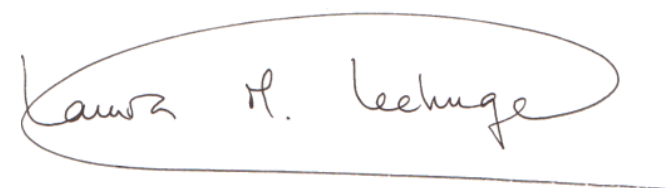

Prof. Laura M. Lechuga

NanoBiosensors and Bioanalytical Applications Group

Catalan Institute of Nanoscience and Nanotechnology (ICN2)

CSIC and The Barcelona Institute of Science and Technology, and CIBER-BBN 
Received 00th January 20xx Accepted 00th January 20xx

DOI : 10.1039/x0xx00000x

www.rsc.org/

\title{
Trends in photonic lab-on-chip interferometric biosensors for point-of-care diagnostics
}

\begin{abstract}
A. B. González-Guerrero, ${ }^{a}$ J. Maldonado, ${ }^{a}$ S. Herranz ${ }^{a}$ and L. M. Lechuga ${ }^{a,{ }^{*}}$
Portable point-of care (POC) devices for in-vitro diagnostics will be a milestone for the achievement of universal healthcare and environmental protection. The main goal is to reach a rapid, user-friendly and highly sensitive portable tool which can provide immediate results in any place at any time while having a competitive cost. Integrated optical (IO) waveguide basedbiosensors are the most suitable candidates to achieve this ambitious objective. They are able to operate in real samples (as blood, urine, wastewater,..) affording relevant sensitivities even under a label-free scheme. In addition, arrays of IO sensors for multiplexed analysis can be integrated in lab-on-chip (LOC) platforms, providing a truly cost-effective fabrication and miniaturization. Among the different IO biosensors, interferometric ones have demonstrated the highest sensitivity for labelfree detection ever reported. Although first interferometric biosensors were shown in the early nineties, they focused mainly on preliminary proof-of-concept studies; only recently the resilient potential of interferometric biosensors as highly advanced POC have firmly emerged. This review provides an overview of the state-of-the art in photonic interferometric biosensors, their main biofunctionalisation routes and their integration in LOC platforms, while maintaining a special focus on the real analytical applications achieved so far.
\end{abstract}

\section{Introduction}

The demand for autonomous and portable detection systems able to analyse extremely low concentrations of analytes in real samples has driven the search for autonomous point-of-care (POC) devices ${ }^{1}$. POC devices can provide instant results under a cost-effective scheme and can enable the sensitive detection of biomarkers, with applications in numerous areas, such as environmental safety, microbiology monitoring, water sanitation, food quality control and clinical diagnosis, among others. For example, POC devices could provide a fast identification of the most spread diseases such as diabetes, stroke, pneumonia, hepatitis A, HIV, malaria or tuberculosis. They can be employed as a screening method for metabolic disorders and infections and can provide support for adherence to treatments. Significantly, POC technologies are opening a window of hope for the economically disadvantaged countries and low-resource environments, where most of the population does not have access to hospitals or clinical laboratories.

Although considerable advances have been achieved in the area of POC devices ${ }^{1}$, few of them are commercially available. Several technical challenges must be still surpassed to finally offer automated, robust, reliable and cost-effective POC devices in order to fully explore their potential impact. Issues as

\footnotetext{
Nanobiosensors and Bioanalytical Applications Group. Catalan Institute of Nanoscience and Nanotechnology (ICN2), CSIC, the Barcelona Institute of Science and Technology and CIBER-BBN, Campus UAB, Ed-ICN2, 08193 Bellaterra, Barcelona, Spain.

† Footnotes relating to the title and/or authors should appear here.

Electronic Supplementary Information (ESI) available: [details of any supplementary information available should be included here]. See DOI: 10.1039/x0xx00000x
}

miniaturization, multiplexing, full-automatization with network connection, microfluidics integration, reagents and bioreceptors drying and store, are still in the pipeline of the POC research and development. However these implementations cannot be supported by analytical technologies currently employed in diagnostics despite of their high precision and sensitivity. The main reason is that standard techniques require complex procedures, large and costly instrumentation, preparation of the samples, and trained personal. Moreover, most of them employ florescent labels or amplification steps, complicating even more the detection procedure.

In this context, biosensors can be considered as a real alternative to current detection technologies to become part of a POC system 2 ; a biosensor directly detects a specific substance from a complex sample by combining a sensitive transducer with a selective bioreceptor within the same device, converting the specific biointeraction into a measurable signal that can be related to the concentration or the presence of the substance under analysis.

Within the large number of existing biosensors, optical ones are able to operate in a label-free pattern since they are surface mass-sensitive; changes of the light (e.g. intensity, wavelength, polarization and phase) can be evaluated when analytes are trapped on the sensor surface through the evanescent field. SPR biosensor has been and still is the most widely employed optical biosensor since it first description in 1983. It has demonstrated an outstanding ability to analyse biological interactions. The exceptionally high number of publications in plasmonic biosensors is a proof of its profound impact, with many and diverse interesting results: characterization of carotenoidbinding proteins ${ }^{3}$, analysis of drug discovery processes, quality 
analysis of pharmaceutical compounds 4 and the characterization of bacterial carbohydrate antigens 5 to mention a few. However, SPR sensor suffers from an intrinsic limited sensitivity which can be only improved by adding secondary recognition elements ${ }^{6}$ at the expenses of losing the advantages of a label-free methodology. Moreover, the relative large size of SPR-based configurations hinders their miniaturisation in POC devices. Later on, Localized Surface Plasmon Resonance (LSPR) biosensors ${ }^{7}$ were suggested as a way to improve the sensitivity while maintaining the advantages of label-free sensing. LSPR also allows for miniaturization and multiplexing, suitable for POC systems and some commercial POC plasmonic systems have emerged ${ }^{8}$. However, the reported sensitivity for LSPR label-free biosensors ${ }^{7}$ is only slightly better than the one achieved by SPR, being not enough for relevant clinical and environmental applications.

For these reasons, sensors based on integrated optics (IO) are attracting increasing attention in the POC diagnostics field. They consist of compact waveguides contained on chips which can be easily miniaturized with the opportunity to fabricate arrays of identical sensors for multiplexed analysis. These IOsensor chips can be easily integrated with a polymer microfluidics and need very low quantities of a sample (few $\mu \mathrm{L}$ ) for the analysis. The sensor-microfluidics integration makes these systems insensitive to vibrations, a fundamental requirement in portable devices. They present other advantages such as robustness, reliability and low power consumption. Furthermore, due to the reduced dimensions of the waveguides (from micrometers to nanometers), several sensors can be produced in parallel in the same wafer meaning a strong potential for mass fabrication, which significantly can reduce the final price of the analysis.

Main working mechanism of 10 biosensors is based on the evanescent wave detection principle (see Fig. 1). Part of the electromagnetic field of the light confined in the core of a waveguide is spread out interrogating a volume of several hundred of nanometers over its surface. Any bimolecular event taking place on the sensor surface (e.g. the interaction of an immobilised bioreceptor with its specific analyte) will change the surface refractive index (from $n_{\text {surface }}$ to $n_{\text {surface }}$ ) modifying the characteristics of the guided light (from $\mathrm{N}_{\text {effective }}$ to $\mathrm{N}^{\prime}$ effective). This change can be quantitatively related to the concentration of the analyte in a sample.
Different IO arrangements have been proposed during the last two decades; most relevant configurations are grating couplers, interferometers, microring resonators, photonic crystals, silicon wire and slot-waveguides based systems 9 . Despite all of them are refractive index sensors operating by the evanescent field principle, the different physical arrangements lead to different quantifiable properties such as the detection limit (LOD), one of the most employed parameter to compare the performance between different sensors. LOD is defined as the minimum variation of refractive index that produces a detectable signal which is considered at least three times higher than the noise of the experimental system. Two different sensitivities can be differentiated: bulk and surface sensitivity. The assessment of the bulk sensitivity (i.e. the change in the refractive index affecting all the volume occupied by the evanescent field) is usually employed to compare the different IO transducers and it is expressed as $\Delta n_{\min }$, in refractive index units (RIU). The surface sensitivity is defined as the variation in the refractive index taking place only in a thin layer in proximity of the sensor surface, where the biomolecular recognition takes place, normally expressed as surface mass density $(\Gamma)$ in pg. $\mathrm{mm}^{-}$ 2, which can be calculated by De Feijter's formula ${ }^{10}$.

Among the different 10 sensors, interferometric devices show the lowest detection limits $\left(10^{-8} \mathrm{RIU}\right.$ for bulk sensing and below $0.01 \mathrm{pg} . \mathrm{mm}^{-2}$ for surface sensing) while maintaining the requirements of miniaturization, low-cost and label-free analysis ${ }^{9,10}$. Since these sensors afford the highest sensitivity, they are the most attractive for POC devices. Different interferometric configurations such as Young, Mach-Zehnder, Bimodal Waveguide interferometers and dual polarization interferometry have been described in the literature. The characteristics of each configuration will be later discussed highlighting their strengths and weaknesses.

However, the final LOD of a biosensor POC device for a particular bioapplication is not only due to the physical transduction mechanism employed but is also highly dependent of the biofunctionalisation protocol. The functionality of the bioreceptor layer is a key step in the final performance of any sensor device; this receptor layer must interact with enough specificity and selectivity with the substance to be detected. For that, bioreceptors must be properly attached to the sensor surface. First step is to chemically provide the inert surface with chemical functional groups, mainly using linking molecules

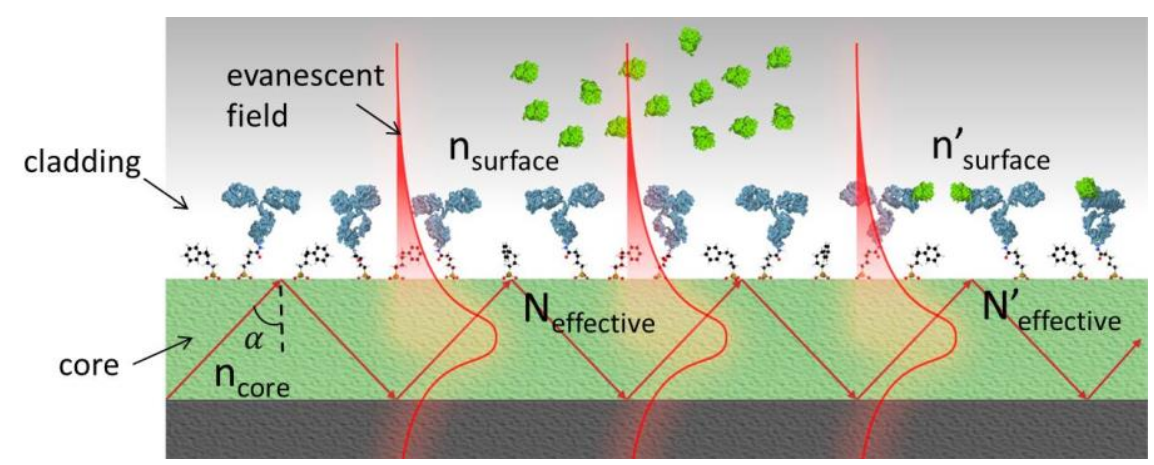

Fig. 1 Evanescent field sensing principle. Part of the light confined in a waveguide is spread out outside the core and interacts with the external medium. When the refractive index of the surface $\left(\mathrm{n}_{\text {surface }}\right)$ changes due to a biointeraction $\left(\mathrm{n}_{\text {surface }}^{\prime}\right)$, the effective refractive index of the waveguide $\left(\mathrm{N}_{\text {effective }}\right)$ is also modified $\left(\mathrm{N}_{\text {effective }}\right)$. 
containing a group reactive to the surface in one end and a functional group reactive to the biomolecule in the other end. Second step is the bioconjugation of the bioreceptor with the ended functional groups of the sensor surface by activating it or by using cross-linkers. In addition, unspecific adsorptions are always expected when real samples are analysed. The most convenient way to reduce such unspecific signals is to design bioreceptor surfaces able to suppress the fouling of unspecific molecules. The chosen blocking method will depend on the matrix complexity of the sample to be analysed (urine, blood, serum, sea water...) being blood and serum the biofluids presenting the highest fouling levels. Unfortunately, there is not a universal method for providing the sensor surface with the appropriate bioreceptor layer and depending on the application, a custom-designed biofunctionalization procedure must be designed.

In this review, we give a critical overview of the different interferometric configuration and their integration in LOC platforms. Second, we discuss different functionalisation routes, bioconjugation and blocking strategies that can be employed to tether the bioreceptors to the transducer surface. Following, we extensively explore the different applications developed so far using interferometric biosensors. Finally, we describe the latest trends in developing truly POC devices using this interferometric technology.

\section{Interferometric biosensors}

\subsection{Working principle of interferometric sensors}

The extraordinary sensitivity of interferometric devices relies on their working principle; the interference of two light waves which are experience different optical path lengths. In this configuration, one of the light waves is interacting with the sample whilst the other one acts as a reference. When both light waves interfere, the resulting light wave experiences a phase variation proportional to the change in the surface refractive index. This phase variation can be related to a bulk refractive index change or to a biomolecular interaction if a previous receptor layer was included in the sensing arm. In interferometry, phase variation is proportional to the interaction length of the sample within the evanescent field. As a consequence, the sensitivity of a particular interferometric configuration can be increased by enlarging the length of the sensor area. In addition, the reference arm of the interferometric devices can be employed to compensate external fluctuations, as for example, the dependence of the refractive index with temperature.

There are two possible configurations for a waveguide interferometric biosensor. The first option is that light waves of the same wavelength and polarization (sensing and reference) propagate by different paths having the same effective refractive index (Neff). This is the most employed configuration; the Mach-Zehnder (MZI), Hartman (HI) and Young interferometers ( $\mathrm{YI}$ ) and the dual polarisation interferometry (DPI) fall into this category. The second option is that light waves of the same wavelength and polarization propagate by the same path having different Neff, as in the case of the Bimodal Waveguide Interferometer (BiMW).

\subsection{Types of interferometric biosensors}

Mach-Zehnder interferometer (MZI). In a MZI device (see Fig. 2a), a polarised and monochromatic light is coupled in a channel waveguide and split by a Y-junction into two light beams. One of the light beams will travel along the reference arm and the other one along the sensing arm interacting with the sample. The exiting two light beams are recombined again by another $Y$ junction; the resulting light will experience a phase variation if a change in the surface refractive index occurs in the sensing path. The phase variation will originate a cosine-type variation in the intensity of the output light that can be read-out by a photodetector or a CCD camera. By using a MZI configuration, extremely sensitive sensors have been achieving obtaining a LOD of $2 \times 10^{-8} \mathrm{RIU}$ and $0.01 \mathrm{pg} . \mathrm{mm}^{-2}$, respectively ${ }^{11}$. In addition, $\mathrm{MZI}$ biosensor has a high level of integration since light splitting and recombination are made on-chip. The drawbacks of this configuration are related to the cosine-type intensity distribution of the interference pattern, which involves: i) a phase ambiguity of the signal due to its periodicity, ii) possible misinterpretation of the signal due to intensity fluctuations of the light source and iii) sensitivity fading when the signal is tuned close to one of the extreme values of the transmission curve.

The Hartman interferometer (HI). The $\mathrm{HI}$ uses slabs waveguides (see Fig. 2b). Light is divided into two beams by a splitter outside the chip and coupled to the slab waveguide via gratings. Then light travels by contiguous regions which are functionalised with specific (sensing path) or nonspecific receptors (reference path). The light output from both regions are outcoupled using gratings and combined by a lens, generating an interferogram in a CCD camera. This device has a LOD in the range of $10^{-6}$ RIU

12. An advantage of this configuration is that the issues related to the sinusoidal signal are overcome by the analysis of the interferogram by fast Fourier transform (FFT). The main limitation relies in the crosstalk effect between sensing and reference beams due to the slab waveguide configuration. In addition, the lower level of integration of the $\mathrm{HI}$ implies a complex mechanical stabilization of the optical components for a future POC platform.

The Young interferometer ( $\mathrm{YI}$ ). In a $\mathrm{YI}$ (see Fig. 2c), light is coupled in a channel waveguide and split by a $\mathrm{Y}$-divisor into two beams; one will travel by the sensor area and the other by the reference waveguide. The output light from both waveguides is projected onto a CCD camera achieving an interferogram which can be analysed by FFT. The best LOD achieved with this device has been $6 \times 10^{-8} \mathrm{RIU}$ and $0.02 \mathrm{pg} . \mathrm{mm}^{-2}$, respectively ${ }^{13}$. The $\mathrm{YI}$ has a high sensitivity and unambiguous signals; however the required distance between the waveguides output and the CCD makes it more prone to vibrations and less suitable for POC integration. Dual polarisation interferometry (DPI). This sensor (see Fig. 2d) uses two slab waveguides where the lower layer is the reference 
a)

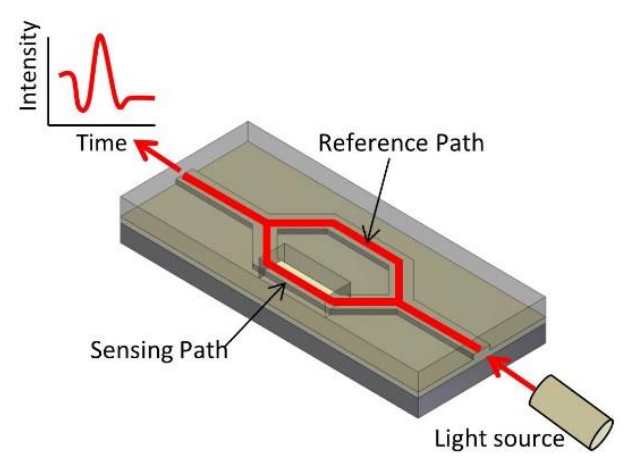

c)

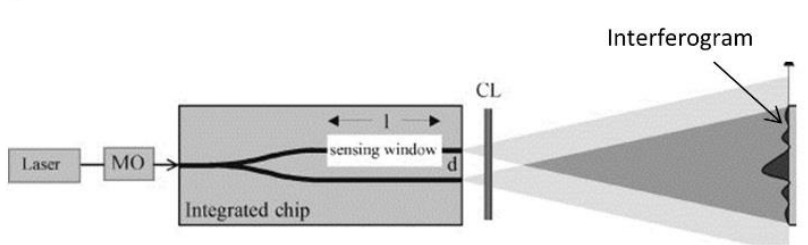

b)
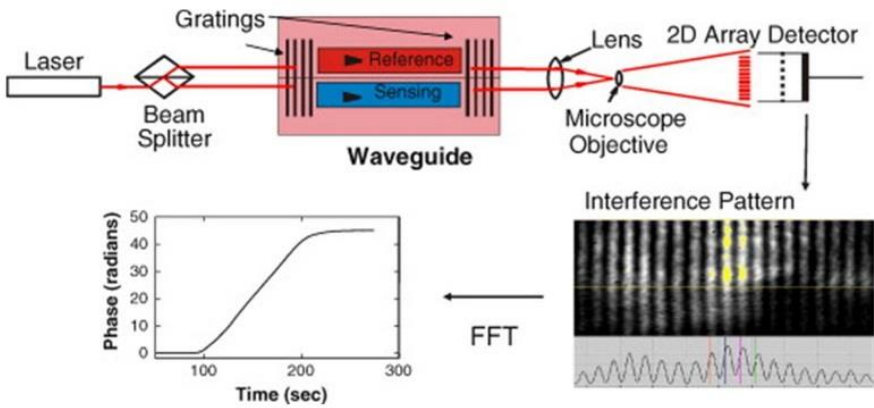

d)
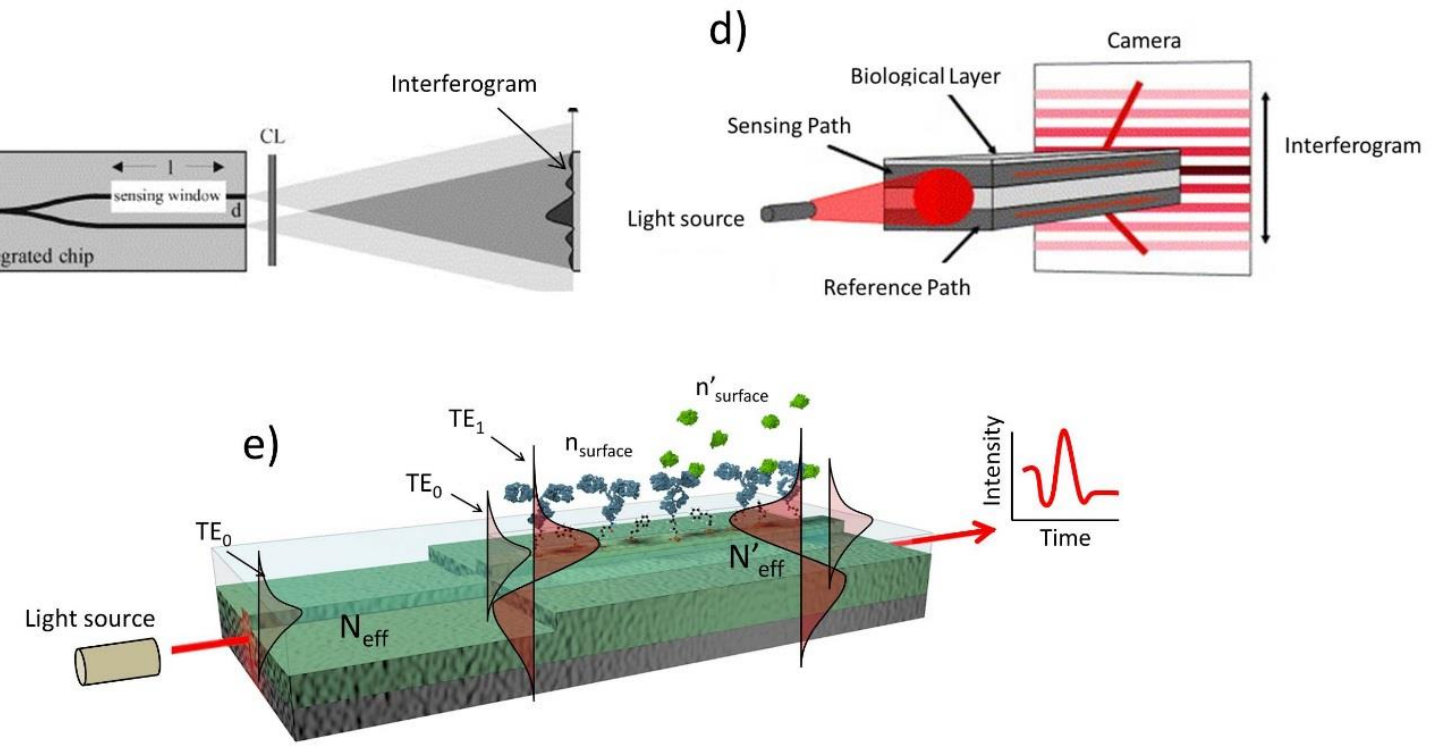

Fig 2 Schematic diagram of: a) Mach-Zehnder interferometric sensor, b) Hartman interferometer (reprinted from [] Copyright (2002) with permission from Elsevier), c) Young interferometer biosensor (reprinted from [] Copyright (2002) with permission from Elsevier), d) dual-polarization interferometer (reprinted from [] Copyright (2004 with permission from Elsevier) and e) Bimodal waveguide biosensor.

waveguide and the upper layer is the sensing one ${ }^{14}$. An additional layer is placed between both waveguides for an effective isolation. The intensity distributions from the "up" and "down" waveguides are projected into a CCD camera obtaining an interferogram. By using a fast liquid crystal switch it is possible to excite the transversal electric (TE) and the transversal magnetic (TM) modes in the waveguides at the same time. The resulting sensor is able to provide real-time information of the biolayer growth obtaining a LOD of $10^{-7}$ RIU and $0.1 \mathrm{pg} \cdot \mathrm{mm}^{-2} 15$. This biosensor was commercialized by the company Farfield from 2000 to 2011.

Bimodal Waveguide interferometer (BiMW). In the BiMW sensor (see Fig. 2e), two modes of light of the same polarization and wavelength but of different order $\left(\mathrm{TE}_{0}\right.$ and $\left.T E_{1}\right)$ travel while interfering in a straight waveguide. For this, light is coupled in a single-mode waveguide in which only $\mathrm{TE}_{0}$ can propagate; after some distance, light is coupled in another waveguide supporting two transversal modes. In the step junction, $\mathrm{TE}_{0}$ and $\mathrm{TE}_{1}$ are excited and both propagate until the end of the chip. $A$ biointeraction will cause a change in the surface refractive index that will affect differently to both modes due to the different distributions of their evanescent fields. Then, the interference of both modes will generate a phase variation producing a sinusoidal variation of the intensity distribution of the light exiting the waveguide ${ }^{16}$. This configuration has demonstrated a LOD of $5 \times 10^{-8} \mathrm{RIU}$ and $2.8 \mathrm{fg} \cdot \mathrm{mm}^{-2}{ }^{17}$. As the MZI configuration, the BiMW device also presents the drawbacks of phase ambiguity and sensitivity fading but solves the misinterpretation of the signal by normalizing the signals output.

To summarize this section, the MZI and the $\mathrm{YI}$ devices consist of channel waveguides which entail a more complex fabrication than the devices based on slab waveguides such as the HI. However, the signal processing of slab waveguides is more difficult for multiplexed detection since parasite light and crosstalk must be minimized. A different interferometric arrangement, the BiMW sensor allows a simpler fabrication process than the $\mathrm{MZ}$ and the $\mathrm{YI}$ since it consists of a straight waveguide which avoids the inclusion of Y-splitters. On the other hand, MZI and BiMW devices provide a high level of integration since splitting and recombination of light are done on-chip which provides a further stabilization to mechanical vibrations. However, the on-chip recombination of the light 
coming from the sensing and the reference arm involves a sinusoidal intensity output which drives to an ambiguous signal. Despite this drawback is not present in the $\mathrm{YI}, \mathrm{HI}$ and DPI configurations which output interferograms are an unambiguous and linear signal; is at the expenses of increasing the complexity of the post-processing of the signal. In addition, these devices have lower level of integration which can be a drawback when considering their incorporation in a POC system.

\subsection{Lab-on-chip integration for POC platforms}

The multiplexed detection of several analytes from the same sample using an array of interferometric sensors can be achieve by incorporating in the same platform: $i$ ) the in/out-coupling of light, ii) the individual microfluidic channels for bringing the sample to each of the sensor areas and iii) an appropriate modulation system for unravelling the interferometric signal and transforming in a linear read-out (only for configurations with intensity output).

Regarding the in/out-coupling of the light, the most employed techniques are prim coupling ${ }^{18,19}$, grating-coupling, end-fire ${ }^{16}$ and butt-coupling ${ }^{20}$ since they show a good performance for laboratory experiments. However, most of them have important limitations for implementing in real POC devices due to poor alignment tolerances. A higher alignment tolerance has been demonstrated for grating assisted coupling 21,22 which is a key point for POC systems in which the biochip must be a disposable element.

The sinusoidal behaviour of the interferometric signal is other issue to be solved before integration An option is including $3 \times 3$ couplers 20,23 and $2 \times 3$ multimode interference coupler 24 However, these solutions increase the complexity of the MZI fabrication process. Alternative approaches developed up to now consist of phase compensation techniques such as electrooptical ${ }^{25}$, thermo-optical ${ }^{26}$ and magneto-optical ${ }^{27}$ modulation as well as the use of liquid crystals ${ }^{28}$. However, these approaches need the use of bulky optical equipment or involve additional fabrication steps. Other interesting possibility is to periodically modulate the incoming wavelength of a common laser diode, introducing a phase variation modification altering the $\mathrm{N}_{\text {eff }}$ of the guided modes. This all-optical modulation method has been successfully applied to the MZI configuration ${ }^{29}$ and recently to the BiMW device ${ }^{30}$; by using FFT for the postprocessing of the signal, a linear and unambiguous response can be provided in real-time.

A different issue is how to bring the liquid sample in contact with the sensor area. Microfluidic systems usually employ are: i) double side adhesive which bonds the chip with a glass or plastic tape including the tubes ${ }^{31}$ or ii) polydimiethylsiloxane (PDMS) channels fabricated by soft lithography ${ }^{32}$. Despite these microfluidic systems have a high versatility, their relatively large width hinders the evaluation of each sensor independently since the minimum separation between waveguides (only few hundred of microns or less) is always considered to reduce fabrication costs. For this purpose, the fabrication of microfluidics channels at wafer level is mandatory for the development of a POC device. Moreover, the reduction of the fluidic channels can result in an enhanced LOD for a particular biapplication by using low sample volumes and by reducing the diffusion process ${ }^{33}$. Despite all these advantages, the integration of the microfluidics with the interferometric sensors at wafer level entails a high technological complexity and few works have been reported ${ }^{13,21}$.

Taking globally these considerations into account, several works have been recently reported demonstrating all of them, good approaches for a final POC integration. A frequencyresolved $\mathrm{MZI}$ biosensor (FR-MZI) in which the conventional monochromatic laser source is replaced by a white-light source has been reported ${ }^{34}$. In this case, the use of white-light excitation, in addition to be cheaper, circumvents the ambiguity and signal fading problems, demonstrating a bulk LOD in the range of $10^{-6}$ to $10^{-7}$ RIU ${ }^{35}$. Later, a broad-band light source was integrated on the sensor which represented a significant progress towards total POC integration. However, this implementation was at the expenses of losing sensitivity $\left(L O D=10^{-5} \mathrm{RIU}\right)$, increasing fabrication complexity and dealing again with sinusoidal signals ${ }^{36}$. Other important POC implementation has been achieved for a MZI device in which the sensor arm consisted of a slot waveguide while the reference arm consisted of a strip waveguide ${ }^{31,37,38}$. In this sensor, light at $1542 \mathrm{~nm}$ was in- and out-coupled by grating couplers achieving a LOD of $1.29 \times 10^{-5}$ RIU. Recently, an asymmetric MZI (aMZI) was proposed in which a small path length difference is added between both arms resulting in a wavelength dependency of the output phase ${ }^{39}$. By scanning the aMZI over a small bandwidth it is possible to obtain directly the phase shift due to a refractive index change. It can be also mention a different MZI configuration in which $850 \mathrm{~nm}$ light is coupled by grating couplers and an on-chip spectrum analyzer (spectral filter) has been incorporated for the read-out of the out-coupling light which consists of arrayed waveguide grating. The LOD of this device was found in the range of $10^{-7}$ RIU. The strong potential of this configuration is that a broadband light can be used as source as opposed to an expensive tunable laser. In addition, a fully integrated MZI configuration is maintained while an interferogram is obtained as output signal. This design could overcome the drawbacks related to intensity signals by directly obtaining the $\Delta \varphi$ using a proper signal postprocessing ${ }^{40}$.

However, the ideal LOC device for POC multiplexed testing must incorporate not only elements for the in- and out-coupling of light as grating couplers, a simple system for signal modulation and microfluidic channels but also a complete sensor array in the same platform . For example, Fig. 3 shows different options to the simultaneous light coupling via gratings for a multiplexed BiMW biosensor. The first one involves the use of several light beams to couple light in a single transducer. The second one requires one light beam illuminating a single grating but then the waveguide must be splitter to distribute the light into the different transducers. The third option is to illuminate all the gratings simultaneously. Finally, the multiplexed readout can be done with a CCD camera. All these configurations have advantages and drawbacks which must be independently 
examined before a final decision for a competitive POC platform can be taken.

\section{Sensor surface functionalisation}

To achieve a specific and sensitive response towards the analyte of interest, a recognition element or bioreceptor must be previously immobilised on the sensor surface. The immobilization procedure will be determined by the material of the sensor surface and the reactive functional groups available in the bioreceptor, and it must preserve the bioreceptor activity, allowing the recognition of analytes while avoiding structural damage ${ }^{41}$. Moreover, the resulting biosurface must be inert enough to other interfering compounds normally present in the sample matrix. This is of high relevance for labelfree optical biosensors in which nonspecific adsorptions can lead to false positive/negative results ${ }^{42}$.

The most common bioreceptors employed in biosensors are antibodies, protein derivatives or nucleic acids ${ }^{43}$. Other biorecognition agents, less commonly employed, are peptides, phages, whole cells, aptamers or mimic inorganic structures such as molecular imprinted polymers. Antibody/antigen pairs have been the most employed system for proof-of concept biosensors both in direct (antibody-coated transducer surface) 44 , indirect (antigen-coated transducer surface) ${ }^{45}$ or competitive assays ${ }^{46}$. They have been applied in many areas including clinical diagnostics, environmental detection of toxic pollutants or food control, to name a few. On the other hand, nucleic acid-based biosensors have been applied for pathogen detection and infectious disease monitoring, mainly using nucleic acid probe hybridization systems. In this case, the sensor detects specific segments of nucleic acid, unique within the organism of interest, by using complementary sequences as bioreceptors.

Different methods can be used to immobilise the

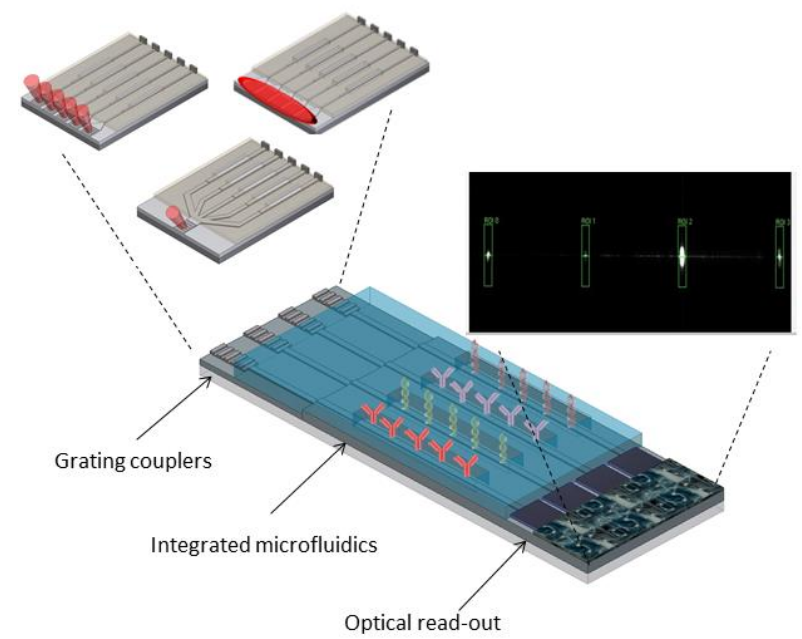

Fig 3 Scheme of the LOC integration of an array of BiMW devices, the microfluidics, different options to incoupling the light on the nanowaveguides and the simultaneous read-out by CCD camera of four different sensors.

bioreceptors on the sensor surface such as physical adsorption, covalent, electrostatic or affinity binding ${ }^{47}$. Although the direct adsorption have been widely used due to its simplicity, it can render in poor reproducibility of the receptor density on the surface, the molecules adopt a random orientation, and they may release from the surface during sample analysis, causing a signal loss ${ }^{48}$. Hence, the most employed method is the covalent bonding ${ }^{41}$.

Different surface modification methodologies for the covalent attachment of the bioreceptors have been described. We will focus our attention on the modification of silicon-based surfaces since they are the main material used for interferometric sensors. In the following, we will discuss the most applied strategies to chemically modify the transducer surface to favour the receptor attachment. We will focus on established methods for bioreceptor binding by direct or crosslinker mediated reactions, with the chemical groups previously introduced on the surface. Finally, we provide a brief summary of the strategies for achieving functionalised low-or non-fouling coated surfaces.

\subsection{Surface chemical modification}

Before bioreceptor attachment, the inert surface material must be chemically modified to introduce functional groups which further facilitate the binding of the bioreceptor. The functionalities mainly applied for the chemical modification of IO transducers are amine $\left(-\mathrm{NH}_{2}\right)$, carboxyl $(-\mathrm{COOH})$, thiol $(-\mathrm{SH})$ or epoxy, which allow the attachment of the receptor to the functional group-ended surface using well-known bioconjugation techniques ${ }^{49}$. Particularly for silicon and silicon nitride materials, the chemical modification strategies can be classified in two main groups: i) chemical modification of the native silicon oxide layer which is always present on these materials, and ii) chemical modification of oxide-free $\mathrm{H}$ terminated surfaces after etching the native oxide layer.

Chemical modification of oxidized surfaces. Most of the methods applied to chemical modification of $I O$ biosensors are based on the molecular grafting of native oxide surfaces, mainly forming selfassembled monolayer (SAM) of alkyl-silanes ${ }^{48}$. This chemistry is not limited to Si-based surfaces; other hydroxylated surfaces such as metal oxide surfaces as aluminum $\left(\mathrm{Al}_{2} \mathrm{O}_{3}\right)^{50}$, titanium $\left(\mathrm{TiO}_{2}\right)^{51}$, or tantalum $\left(\mathrm{Ta}_{2} \mathrm{O}_{5}\right)^{22}$ can be modified as well by this methodology.

The oxide layer has hydroxyl moieties $(-\mathrm{OH})$ on its surface that can condense with the siloxanes of an organosilane, forming the alkyl-silane SAM. However, the density of reactive hydroxyl groups on the native oxide layer surface is usually low due to the condensation of silanol groups $(\mathrm{Si}-\mathrm{OH})$ to form more stable siloxane groups ( $\mathrm{Si}-\mathrm{O}-\mathrm{Si}$ ) over time ${ }^{52}$. The most effective oxidation techniques for obtaining hydroxyl groups on siliconbased surfaces are oxygen plasma ${ }^{53}$, piranha solution (mixture of concentrated sulfuric acid and hydrogen peroxide at ratios from 3:1 to 7:3) ${ }^{54}$ and $U V / \mathrm{O}_{3}{ }^{55}$, although other oxidants such as sodium hydroxide ${ }^{56}$, nitric acid ${ }^{57}$ and mixtures of ammonium chloride/hydrogen peroxide and hydrochloric acid/hydrogen peroxide ${ }^{58}$ can be employed. 
The silanisation procedure is carried out by immersing the hydroxylated surface into a solution of an alkyl-silane (wet chemistry method) or by exposing the surface to a stream of alkyl-silane gas generated with pressure reduction and/or heat (vapour-phase method). Several parameters as silane concentration, type of solvent (in wet chemistry method), pressure (in vapour-phase method), temperature and reaction time, have a strong influence on the properties (availability of functional groups, thickness and density) of the resulting alkylsilane layer. For example, when working with the amino-ended silane 3-aminopropyltriethoxysilane (APTES), the most widely organosilane used for biosensors, experimental wet chemistry methods employ from anhydrous organic solvents (such as dry toluene ${ }^{59}$, dry acetone ${ }^{60}$ or dry ethanol ${ }^{61}$ ) to hydro-organic mixtures ${ }^{62}$ or even water ${ }^{63}$. Other alkyl chain organosilanes used for biofunctionalisation purposes are carboxyethylsilanetriol sodium salt (CTES, carboxylic acid-ended surface) ${ }^{64}, 3$-glycidyloxypropyltrimethoxysilane (GOPTS, epoxyended surface) ${ }^{65},{ }^{66}$, isocyanatepropyltriethoxysilane (ICPTS, isocyanate-ended surface) 46,67 or (3mercaptopropyl)trimethoxysilane (MPTMS, thiol-ended surface) ${ }^{68}$. The main employed silanes are schematized in Fig. 4.

Due to the simplicity of the method, silanisation has been widely employed for functionalization of silicon surfaces. However has several drawbacks as the unwanted polymerization of the silane molecules which can result in thick inhomogeneous films on the surface ${ }^{69}$, which could affect the performance of the device and the reproducibility of the detection.

Chemical modification of oxide-free $\mathbf{H}$-terminated surfaces. The problems of homogeneity and stability of silanes, related to the polymerization and hydrolysis of $\mathrm{Si}-\mathrm{O}-\mathrm{Si}$, stimulated the search of alternative methods for grafting functional molecules on oxide-free Si surfaces ${ }^{70}$. For these alternative methodology, the native oxide layer is first removed with concentrated HF to achieve a hydrogenterminated surface. To remove the etch products and to clean the surface, HF is usually displace with isopropyl alcohol or other nonoxidant solvent, avoiding milli-Q water rising which may oxidize again the surface ${ }^{71}$. Finally, the surface is modified either by indirect substitution of $\mathrm{H}$-termination by functional organic molecules (such
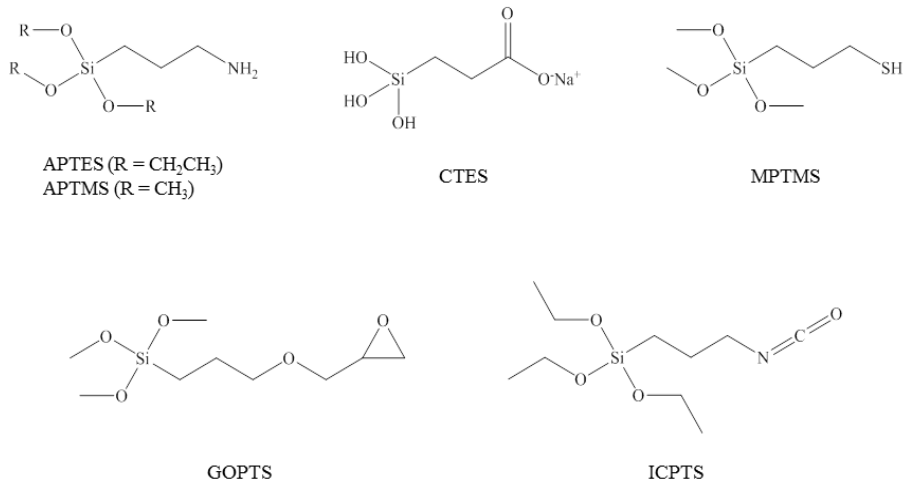

Fig. 4 Most commonly silanising agents used for chemical modification of silicon-based sensor surfaces. as hydrosilylation) or by direct substitution by other atoms or small functional groups.

Despite the number of surface modification protocols based on $\mathrm{H}$-terminated described in the literature ${ }^{72-74}$, their implementation for biosensor applications has been until now very limited. An example is the work of Gooding et al. which describes the formation of carboxylic acid-terminated monolayers in porous silicon (PSi) by thermal hydrosilylation to develop a label-free protease biosensor ${ }^{75},{ }^{76}$. Alternatively, photochemical hydrosilylation was described by De Stefano et al. for the development of DNA PSi-based optical biosensors 77 and by Maquieira et al., who developed an oxide-free silicon nitride surfaces derivatization approach by directly bonding glutaraldehyde to surface through $\mathrm{N}-\mathrm{H}$ bonds ${ }^{78}$, later applied to microring resonator-based biosensors ${ }^{79}, 80$.

It must be noted that despite they achieve more stable, packaged and reproducible monolayers, the functionalisation procedures based on oxide-free surfaces modification entail a high complexity when compared with standard silanisation methods. They require strict anhydrous conditions, the use of halogenated solvent and dangerous chemicals such as HF and $24 \mathrm{~h}$ reactions in refluxing conditions under inert atmosphere.

\subsection{Bioreceptor immobilisation}

The immobilization of the receptor is usually done by covalent binding onto the previously chemically modified sensor surface. The strategy employed will depend on the functional group present on the surface and the functional group available on the bioreceptor for the bioconjugation, being amino groups the most frequently employed for both.

The most common chemistry for covalently immobilised bioreceptors onto amine-ended surfaces is based on $\mathrm{N}$ hydroxysuccinimide (NHS) esters which can react with primary and secondary amines to create stable amide and imide linkages, respectively ${ }^{49}$. However, the preferred cross-linker molecule to activate amino-modified supports with 


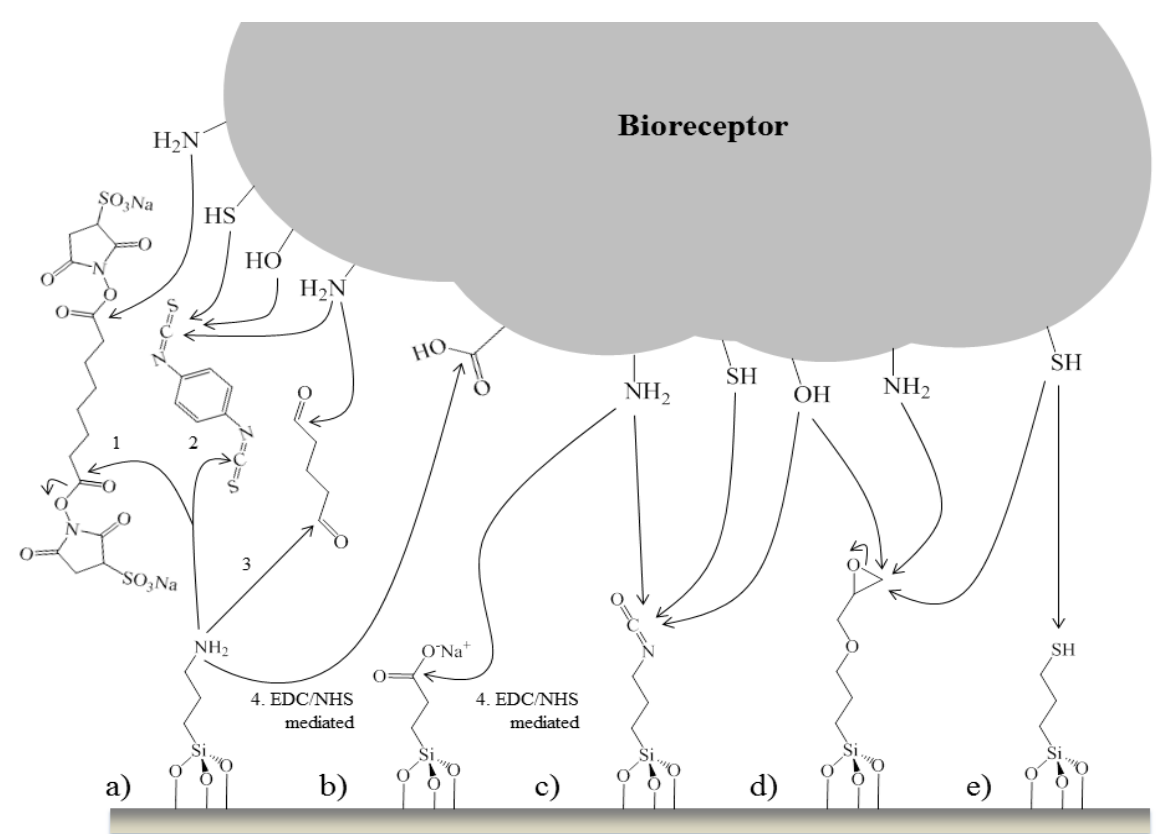

Fig. 5 Bioconjugation procedures employed for: a) amine-, b) carboxy-, c) isocyanate-, d) epoxy-, and e) thiol-ended surfaces. 1) BS3-crosslinking, 2) PDITC-crosslinking, 3) glutaraldehyde-crosslinking, and 4) EDC/NHS-mediated approaches.

isothiocyanate functional groups is $p$-phenylene diisothiocyanate (PDITC). PDITC has the potential to form wellorganized assemblies driven by $\pi-\pi$ stacking, significantly decreasing non-specific binding ${ }^{81}$. Glutaraldehyde has been widely employed but it has some undesirable effects, such as the formation of several layers that could break during the biorecognition process giving inaccurate signals.

In the case of carboxylate-ended surfaces, they can be functionalised with amine-ended bioreceptors after reaction of carboxylic acid and NHS in the presence of $N$-Ethyl- $N^{\prime}-(3-$ dimethylaminopropyl)carbodiimide (EDC) and used to couple amine-containing bioreceptors ${ }^{64}$.

Bioconjugation of biomolecules to epoxide rings involves nucleophilic primary amines, sulfhydryls groups, hydroxyl groups ${ }^{41}$ to create secondary amine, thioether, or ether bond respectively. Finally, thiol-modified surfaces have also been described in the literature for attachment of thiolated oligonucleotides by disulfide bond ${ }^{68}$, or aminated biomolecules (antibodies), previously using heterobifunctional cross-linker mmaleimidobenzoyl-N-hydroxysuccinimide ester (MBS) ${ }^{82}$. A summary of all these bioconjugations techniques can be found in Fig. 5.

\subsection{Avoiding non-specific adsorptions}

For POC devices which must operate in real emplacements and with crude samples, it is mandatory that the sensor surface is resistant to nonspecific adsorption of other molecules in order to avoid false results. In interferometric biosensors, reference waveguides can be used to subtract unspecific signals from the net signal by functionalizing them in the same way that the sensing waveguide ${ }^{83}$. Using this approach in a Hartman interferometer, it was demonstrated the real-time detection of an hormone in serum ${ }^{65}$ and in undiluted whole human blood ${ }^{84}$.
However, unspecific adsorptions could block the binding sites of the immobilized bioreceptors, limiting the receptor/target interaction and, consequently, reducing the sensitivity. In addition, the fouling effect in both waveguides could not be comparable since different bimolecular layers could give rise to different amounts of unspecific adsorption ${ }^{85}$. The use of blocking compounds usually employed in ELISA tests, such as proteins (BSA, fish gelatin or casein) or Tween are not always working well for plasma or serum blood evaluations using labelfree biosensors. For that, the development of proper nonfouling surfaces has been in the research focus. Ideally, lowfouling surfaces for biosensing purposes must provide a quantity of non-specific biomolecules adsorbed below the LOD of the bioapplication ${ }^{42}$.

Hydrogen bonds, ionic, polar and hydrophobic interactions are the main driving forces causing nonspecific adsorption of biomolecules on surfaces. As a consequence, the treatment of the surface with reagents to increase its hydrophilicity has been extensively described to reduce hydrophobic interactions. This is the case of poly(ethylene glycol) (PEG) and its derivatives which are the most common polymers employed for nonfouling applications ${ }^{86}$. Thereby, oligo(ethylene glycol) (OEG) alkanethiols incorporating a functional group such as $-\mathrm{COOH}$ (HS-OEG-COOH) or-OH (HS-OEG-OH) have been widely used for SPR biosensing ${ }^{87}$. Recently, PLL-PEG has been employed to passivate the glass surrounding gold-nanodisks in a LSPR biosensor significantly reducing non-specific binding ${ }^{88}$. In this direction, poly(ethylene oxide) (PEO) have been employed for binding on low fouling amounts from blood plasma and serum, respectively ${ }^{89}$. Interestingly in this work, the PEO/Dopaminemelanine layers were growth on silicon oxide surfaces and provided the surface with available amino groups suggesting 
that this functionalisation could be employed for silicon-based biosensors.

Electroneutral surfaces made by zwitterionic materials could also avoid protein adsorption by a strong hydration via ionic solvation ${ }^{90}$. This is the case of surfaces covered by a nonfouling polymer as the poly(carboxybetaine methacrylate) (polyCBMA) ${ }^{91}$. In addition, this compound provides a high quantity of functional groups for the attachment of biomolecules. Another CBMA-based material was developed by including 3,4-dihydroxy-L-phenylalanine (DOPA), in which two DOPA-poly(carboxybetaine methacrylate) (DOPA ${ }_{2}-$ pCBMA $\left._{2}\right)$ were linked to increase the polymer surface density ${ }^{92}$. More interesting for interferometric biosensors, the $\mathrm{DOPA}_{2}-\mathrm{pCBMA}_{2}$ has been employed for the functionalisation of a $\mathrm{SiO}_{2}$-coated SPR chip via metal-oxygen coordination complex formed between DOPA and the oxide surface, following by bioconjugation with an antibody ${ }^{93}$.

Although considerable advances have been obtained in the development of non-fouling surfaces using PEG and CBMA derivatives, the achievement of zero unspecific adsorption at the sensitivity level presented by interferometric biosensors is still a challenge.

\section{Real applications}

Considering the outstanding sensitivity, potential multiplexing, low cost and integration capabilities of interferometric 10 biosensors, we can envisioned their use for advanced liquid biopsies, blood test for detection circulating tumour cells (CTCS), detection of mutated DNA fragments or of biomarkers liberated to the bloodstream due to the onset of an illness before any symptom, among other relevant clinical applications. In the case of environmental control, the sophisticated and expensive methodologies currently employed to detect contaminants which present significant harmful effects on the human and animal health using chromatographic techniques and/or mass spectrometry detection could be replaced by a few minutes assay by introducing the sample in an interferometric POC system. The simultaneous detection of several analytes, biomarkers or toxins in the same sample is other revolutionary aspect that could be afforded by the interferometric POC technology. For example, in the clinical practice is well-known that a panel of biomarkers can improve the predictive performance over individual markers.

One important advantage of the interferometric $\mathrm{POC}$ system is the reduction of the analysis price which will enable the extension of screening tests for the early detection of diseases to a wider range of population. The possibility to place autonomous POC devices in primary health care units or even at home, could save costs as compare to the current situation, where most of the analysis are done in specialised, centralised laboratories.

\subsection{Clinical diagnostics}

Most of the biomarkers in human biofluids as blood or urine are normally present in very low concentrations, well-below the $\mathrm{ng} / \mathrm{mL}$ level, which hinders their label-free detection. Interferometric biosensors can afford a label-free detection in real-time for such low values of concentration. For example, with a BiMW we have detected with a high level of sensitivity, the human Growth Hormone ( $\mathrm{hGH}$ ), an important biomarker for the diagnosis of acromegaly and other growth disorders. The transducer surface was functionalised via CTES silanisation ${ }^{32}$. The calibration curve for the detection of hGH samples in buffer solution using a competitive immunoassay is shown in Fig. 6 . The LOD was only of $8 \mathrm{pg} \cdot \mathrm{mL}^{-1}$, three magnitude orders better than the one obtained with a SPR biosensor ( $\left.4 \mathrm{ng} \cdot \mathrm{mL}^{-1}\right)$ using the same immunoreagents ${ }^{94}$. This result indicates the feasibility of detecting a particular protein at physiological range in a labelfree way using interferometry.

Interferometry is also adequate for detecting proteins in complex samples. In a recent work, the BiMW biosensor performance was demonstrated for the detection of hGH in

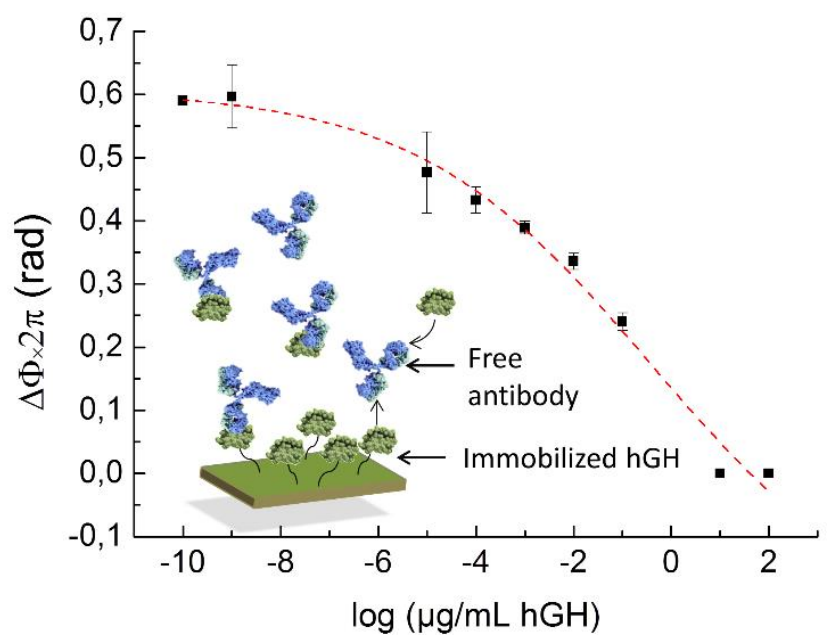

Fig. 6 Calibration curve for the detection of hGH in buffer by a competitive immunoassay using a BiMW interferometric biosensor. Inset: scheme of a competitive immunoassay

undiluted urine samples using a direct immunoassay format ${ }^{17}$. In this case, to avoid non-specific adsorption, sensors were silanised using APTES and the specific antibody was immobilised using a PDITC cross-linker. The biosensor showed a LOD of only 3 pg. $\mathrm{mL}^{-1}$, without the need of any sample pretreatment or amplification steps, proving the label-free detection of hGH at a range of concentrations relevant to clinical diagnostics in urine (pg. $\mathrm{mL}^{-1}$ range).

The development of autonomous POC devices would have a high impact in developing countries where health care systems are not well developed. A prime example is the case of the diagnosis of tuberculosis (TB) infection which has the highest incidence in countries with low per capita income, and whose detection in early states could prevent its spread. Mycobacterial culture is now the laboratory standard for the diagnosis of active TB, but it is costly and takes weeks to provide the results. Interested in solving these issues, Nagel et al. published ${ }^{22}$ a hybrid YI to detect TB-specific antibodies in blood serum of infected persons. Sensor chips were silanised with APTES and activated using PDITC to couple the 38-kDa antigen employed as bioreceptor. Although the LOD of the sensor was not 
determined, they demonstrated that it was possible to diagnose TB-infected patients from their blood samples in a label-free way. In the context of TB diagnosis, the European project POCKET 40 is developing a low-cost Point-Of-Care test for Tuberculosis detection using an fully-integrated MZI interferometric approach. The goal of this project is to develop a rapid and accurate diagnostic tool for TB diagnosis to be applied in resource-constrained settings. The combined detection of Lipoarabinomannan (LAM), a bacterial product, and Ag85 antigen in urine samples using a POC system is proposed to increase the specificity of current flow-lateral tests $(60 \%)$ by a direct immunoassay.

Until the development of the PCR in the 1990s, virus detection methods consisted of culture, antigen detection and serology, yielding low overall virus detection rates ${ }^{95}$. Although PCR is accurate and sensitive, it does not allow a real-time detection and has associated a high cost To overcome these drawbacks, the detection of herpes simplex virus type 1 (HSV-1) was demonstrated by using a multiplexed $\mathrm{YI}$ developed by $\mathrm{MESA}^{+}$Institute ${ }^{96}$. Three of the four YI channels were employed for immobilizing different bioreceptors, demonstrating the possibility of measuring multiple analytes from the same sample, while the other channel was used as reference. For the immobilisation of the antibodies, the sensor areas were functionalised by physically adsorbing protein $\mathrm{A}$. They were able to detect HSV-1 at a concentration of 850 particles. $\mathrm{mL}^{-1}$, claiming a LOD close to single virus detection. The detection of HSV-1 in serum samples was demonstrated despite a background due to unspecific proteins.

During last years, microRNA (miRNA), small noncoding RNA molecules that regulate gene and protein expression and which circulate in human biofluids, have emerged as potential biomarkers for the diagnosis of several diseases including cardiovascular diseases ${ }^{97}$, hepatitis $\mathrm{C}$ infection ${ }^{98}$ and cancer ${ }^{99}$. Hence, there is a strong interest in developing POC devices for detecting miRNA in a direct, fast and high sensitive way. Liu et al. ${ }^{31}$ detected several miRNAs (miR-21 and let-7A) in urine samples of bladder cancer patients by using a slot-MZI POC system. DNA receptors were immobilised following a functionalising with APTES. They achieved a LOD of fmol. $\mu \mathrm{L}^{-1}$ (equivalent to $\mathrm{nM}$ level). Despite the limited sensitivity, the miRNAs were detected without amplification from clinical urine samples and results were validated by PCR analysis.

Recently BiMW biosensors have demonstrated ultrasensitive detection of microRNAs in complex media ${ }^{100}$. Concretely, the BiMW biosensor was employed for the detection and quantification of miR-181a at attomolar concentrations (LOD = $23 \mathrm{aM})$. The extreme selectivity of the biosensor was demonstrates by showing the discrimination of homologous sequences at the level of single nucleotide mismatch. The biosensor was employed for the direct evaluation in human urine samples of bladder cancer patients with no need for prior sample purification or amplification steps. A significant overexpression of miR-181a in bladder cancer patients was appreciated when compared with healthy controls, suggesting the implication of this miRNA in bladder cancer. These results showed that the BiMW biosensor can be used as an ultrasensitive and specific diagnostic tool by the early and fast detection and quantification of microRNAs for the prediction of diseases (such as cancer) with well-defined microRNA signatures.

\subsection{Environmental control}

Examples in the environmental area includes the detection of 2,4,6-trinitrotoluene (TNT) in air using the modified-Hartman sensor ${ }^{101}$ with a molecularly imprinted sol-gel as receptor layer. Detection limits for the selective detection of TNT were found in the low parts-per-trillion (equivalent to $\mathrm{pg} \cdot \mathrm{mL}^{-1}$ ). Regarding the on-line monitoring of water quality, a sensor grid based on MZI devices, the EventLab systems by Optiqua Technologies Pte $L t d$, has been implemented in one of the largest drinking water company of The Netherlands. The sensor system (with a bulk LOD in the $10^{-7}$ RIU range) includes a high density grid of integrated $\mathrm{MZI}$ devices placed along the distribution network, which measured the changes in the water refractive index, turning out as an economically viable early warning system. Using the same MZI device, they have recently developed and validated the MiniLab ${ }^{T M}$ system ${ }^{102}$ for detection of Bisphenol $A$ in treated water by a competitive immunoassay. Bisphenol $A$ is a harmful endocrine disruptor employed in the manufacture of plastic materials such as water bottles and food storage containers, which can migrate from the packaging to the food or drinking water. In this biosensor, immobilisation of Bisphenol A on the sensor area was done by functionalising the surface with GOPTS silane and further modification with an aminodextran. Results demonstrated a working range from 0.5 to $5 \mu \mathrm{g} \mathrm{L}^{-1}$ achieving qualitative results by setting a limit of $50 \%$ normalized response as a cut-off to distinguish between "positive" and "negative" samples. In addition, the authors claim that the sensor can be reused for more than 200 cycles over a 6 months period. Regarding the detection of toxic heavy metals ions, it has been demonstrated the real-time detection of $\mathrm{Hg}^{2+}$ in water using a DPI with a T-rich oligonucleotide as bioreceptor ${ }^{103}$. Immobilisation of the receptor was done by assembling a highly charged poly(ethylenimine) (PEI) layer on the oxidized surface and allowing the negatively charged receptor to adsorb on the surface. The LOD was $27 \mathrm{nM}$ which is in accordance with the guideline value set by the World Health Organization (WHO) for $\mathrm{Hg}^{2+}$ in drinking water $(30 \mathrm{nM})^{104}$.

Other interesting example of environmental application is the EU project BRAAVOO 105 focused on the development of Biosensors, Reporters and Alga Autonomous Vessels for Ocean Operation. In this project, a combination of three types of biosensors are used to detect a number of marine priority pollutants (e.g. algal toxins, heavy metals, organic compounds related to oil and antibiotics) as well as biological effects (i.e. general toxicity and stress response). The three biosensors were first developed as standalone modules and further integrated into a buoy and an autonomous unmanned surveying vessel, allowing in-situ multianalyte detection in the ocean media. Periodic information about the concentration of the monitored contaminants will be transmitted via wireless. In this project, the BiMW biosensor is being used as a multiplexed 
immunosensor to assess six specific marine priority pollutants: Irgarol 1051, pentabromodiphenyl ether (BDE-47), domoic acid, okadaic acid, tetracycline and ampicilline). The biosensor shows LODs at $\mu \mathrm{g} . \mathrm{L}^{-1}$ or ng. $\mathrm{L}^{-1}$ levels, depending on the chemical target.

\section{Towards a portable point-of-care device}

The College of American Pathologists defines POC testing (POCT) as tests designed to be used at or near the site where the patient is located, that nor require permanent dedicated space, and that are performed outside the physical facilities of the clinical laboratories. In addition to this definition, an ideal POC device must be autonomous, i.e. samples must be introduced into the device and the outcome must be obtained without further pretreatments in order to be employed by untrained persons.

To directly analyse real samples in a direct and label-free way by optical biosensors the non-specific adsorption must be minimized. Blood samples present an added difficulty since the high content of cells could interfere in the analysis and the achievement of functionalized surfaces for interferometric biosensors presenting zero adsorption is still a challenge. To date, removal of the cellular elements of blood have been achieved by filtration and centrifugation techniques which implies time and costs in medical laboratories. Hence, many research groups have developed different alternatives which could be integrated in a POC diagnosis system. The most popular method is based on microfluidic channels containing a bifurcating region in which the cells are separated into the branch with less resistance to flow and a faster flow rate (the named Zweifach-Fung effect) ${ }^{106}$. However, blood must be diluted with PBS to reduce the concentration of cells which could hinder the detection of low-content proteins. Sedimentation based systems have been also reported to efficiently extracts blood plasma without diluting the sample 107. Nevertheless, platelets were found in the extracted plasma since they sediment at much lower rates. In a recent publication, a continuous flow plasma/blood separator consisting of a $\mathrm{SiO}_{2}$ pillar structure with a size gap of less than a micron was described ${ }^{108}$. Advantages are that it could be compatible with lithographic fabrication process of integrated optical biosensors and that the design of the filter was found to avoid blood-clogging issues.

Another important issue in the case of bacterial and virus identification using biosensors is the necessity of expose their nucleic acids to the sensor. For that, bacterial outer membranes and viral envelopes must be lysed and DNA or RNA must be sheared into an appropriate fragment size. Chemical methods commonly used break the membrane by adding detergents or surfactants following by DNA purification. However, since this procedure is complicated it is difficult to include in a POC platform. Alternatively, sonication can be used to disrupt cellular membranes and spore coat without adding any chemical to the sample which could later interfere with the biomolecular recognition process. However, sonicator devices are bulky and highly expensive and cannot be integrated in a POC device. To overcome this, different small-size sonicators have been proposed, either for real-time disruption of bacterial cells ${ }^{109}$ or for fragmentation of DNA ${ }^{110}$. In both cases, standard materials were employed and the devices could be integrated in POC diagnosis systems.

Other requirements of an autonomous and low-cost POC system are the development of integrated capillary pumps and microfluidic valves to avoid costly flowing systems, the connections with the macroscopic world, the packaging and the storage conditions of individual cartridges chips and the wireless connection with mobile phones, doctor offices or central data stations.

\section{Conclusions and outlook}

This review tries to give a complete overview of the main key aspects in the development of portable devices using interferometric biosensors, from the different available transducers to the development of POC systems, going through functionalisation techniques and highlighting the more advanced bioapplications achieved so far. The different interferometric configurations have been briefly introduced focusing on different factors: i) to obtain the highest sensitivity, ii) to reduce the complexity of the fabrication process, iii) to solve the drawbacks associated with the sinusoidal interferometric signal and iv) to achieve the highest level of integration. We have described the level of development of the different components required for light in-coupling, microfluidics and modulations systems. All those are recently mature technologies that will allow the integration of the interferometric transducers in final POC platforms.

By using interferometric biosensors it has been demonstrated the improvement in the sensitivity, for example of three order of magnitude as compared to SPR biosensor for the same application and without amplification steps. We have also shown the direct detection of $\mathrm{hGH}$ in undiluted urine at relevant physiological levels (few pg. $\mathrm{mL}^{-1}$ ). In addition, direct detection of herpes simplex virus by a YI, miRNA in urine from bladder cancer patients by a slot-MZI and diagnosis of TB by detecting autoantibodies in blood serum have been demonstrated for clinical diagnosis. Regarding environmental control, detection of TNT in air by a Hartman interferometer Bisphenol $\mathrm{A}$ in water using a MZI and $\mathrm{Hg}^{2+}$ in water using DPI have been achieved at relevant concentrations.

Different solutions have been proposed to overcome the limitations of development autonomous POC devices. For instance, a filter can be integrated on-chip to minimize the issues related to the nonspecific signals for blood or other complex biofluids and DNA contained in microorganisms can be extracted by incorporating a microsonicator.

The level of development achieve by the interferometric technology clearly illustrate the huge potential of interferometric biosensors to become part of POC systems in the near future for advanced on-site bioapplications.

\section{Acknowledgements}


J. Maldonado acknowledges the Mexican National Council for Science and Technology (CONACYT) for a doctoral grant. The authors acknowledge the financial support from EPISENS Project of Spanish Ministry of Economy and Competitiveness (TEC2012-34280). The nanoB2A is a consolidated research group (Grup de Recerca) of the Generalitat de Catalunya and has support from the Departament d'Universitats, Recerca i Societat de la Informació de la Generalitat de Catalunya (2014 SGR 624). ICN2 is the recipient of Grant SEV-2013-0295 from the "Severo Ochoa Centers of Excellence" Program of Spanish MINECO.

\section{Notes and references}

1. S. K. Vashist, P. B. Luppa, L. Y. Yeo, A. Ozcan and J. H. T. Luong, Trends in Biotechnology, 2015, 33, 692-705.

2. A. P. F. Turner, Chemical Society Reviews, 2013, 42, 31843196.

3. P. P. Vachali, B. X. Li, A. Bartschi and P. S. Bernstein, Arch. Biochem. Biophys., 2015, 572, 66-72.

4. A. Olaru, C. Bala, N. Jaffrezic-Renault and H. Y. Aboul-Enein, Crit. Rev. Anal. Chem., 2015, 45, 97-105.

5. B. Brogioni and F. Berti, MedChemComm, 2014, 5, 10581066.

6. M. L. Ermini, S. Mariani, S. Scarano and M. Minunni, Biosens. Bioelectron., 2014, 61, 28-37.

7. M. C. Estevez, M. A. Otte, B. Sepulveda and L. M. Lechuga, Analytica Chimica Acta, 2014, 806, 55-73.

8. LamdaGen, http://lamdagen.com/).

9. M. C. Estevez, M. Alvarez and L. M. Lechuga, Laser \& Photonics Reviews, 2012, 6, 463-487.

10. P. Kozma, F. Kehl, E. Ehrentreich-Förster, C. Stamm and F. F. Bier, Biosensors and Bioelectronics, 2014, 58, 287-307.

11. P. Lambeck V., Measurement Science and Technology, 2006, 17, R93.

12. N. F. Hartman, J. Cobb and J. G. Edwards, SPIE - The International Society for Optical Engineering, 1999.

13. A. Ymeti, J. S. Kanger, J. Greve, G. A. J. Besselink, P. V. Lambeck, R. Wijn and R. G. Heideman, Biosensors and Bioelectronics, 2005, 20, 1417-1421.

14. M. J. Swann, L. L. Peel, S. Carrington and N. J. Freeman, Anal Biochem, 2004, 329, 190-198.

15. J. Escorihuela, M. Á. González-Martínez, J. L. López-Paz, R. Puchades, Á. Maquieira and D. Gimenez-Romero, Chemical Reviews, 2015, 115, 265-294.

16. K. E. Zinoviev, A. B. Gonzalez-Guerrero, C. Dominguez and L. M. Lechuga, Lightwave Technology, Journal of, 2011, 29, 1926-1930.

17. A. B. González-Guerrero, J. Maldonado, S. Dante, D. Duval and L. M. Lechuga, unpublished work.

18. D.-f. Lu, Z.-m. Qi and R.-p. Liu, Sensors and Actuators B: Chemical, 2011, 157, 575-580.

$19 . \quad$ Z. M. Qi, S. Zhao, F. Chen, R. Liu and S. Xia, Opt Express, 2010, 18, 7421-7426.

20. P. Hua, B. Jonathan Luff, G. R. Quigley, J. S. Wilkinson and K. Kawaguchi, Sensors and Actuators B: Chemical, 2002, 87, 250-257.

21. D. Duval, A. B. Gonzalez-Guerrero, S. Dante, J. Osmond, R. Monge, L. J. Fernandez, K. E. Zinoviev, C. Dominguez and L. M. Lechuga, Lab on a Chip, 2012, 12, 1987-1994.
22. T. Nagel, E. Ehrentreich-Förster, M. Singh, K. Schmitt, A. Brandenburg, A. Berka and F. F. Bier, Sensors and Actuators B: Chemical, 2008, 129, 934-940.

23. B. Drapp, J. Piehler, A. Brecht, G. Gauglitz, B. J. Luff, J. S. Wilkinson and J. Ingenhoff, Sensors and Actuators B: Chemical, 1997, 39, 277-282.

24. R. Halir, L. Vivien, X. Le Roux, D. X. Xu and P. Cheben, Photonics Journal, IEEE, 2013, 5, 6800906-6800906.

25. R. G. Heideman and P. V. Lambeck, Sensors and Actuators B: Chemical, 1999, 61, 100-127.

26. V. Passaro, F. Magno and A. Tsarev, Opt. Express, 2005, 13, 3429-3437.

27. B. Sepúlveda, G. Armelles and L. M. Lechuga, Sensors and Actuators A: Physical, 2007, 134, 339-347.

28. P. Kozma, A. Hámori, S. Kurunczi, K. Cottier and R. Horvath, Sensors and Actuators B: Chemical, 2011, 155, 446-450.

$29 . \quad$ S. Dante, D. Duval, B. Sepúlveda, A. B. González-Guerrero, J. R. Sendra and L. M. Lechuga, Opt. Express, 2012, 20, 7195-7205.

30. S. Dante, D. Duval, D. Fariña, A. B. González-Guerrero and L. M. Lechuga, Laser \& Photonics Reviews, 2015, 9, 248255.

31. Q. Liu, Y. Shin, J. S. Kee, K. W. Kim, S. R. Mohamed Rafei, A. P. Perera, X. Tu, G.-Q. Lo, E. Ricci, M. Colombel, E. Chiong, J. P. Thiery and M. K. Park, Biosensors and Bioelectronics, 2015, 71, 365-372.

32. A. B. González-Guerrero, M. Alvarez, A. G. Castaño, C. Domínguez and L. M. Lechuga, Journal of Colloid and Interface Science, 2013, 393, 402-410.

33. N. S. Lynn, H. Sipova, P. Adam and J. Homola, Lab on a Chip, 2013, 13, 1413-1421.

34. M. Kitsara, K. Misiakos, I. Raptis and E. Makarona, Optics Express, 2010, 18, 8193-8206.

35. K. Misiakos, I. Raptis, A. Salapatas, E. Makarona, A. Botsialas, M. Hoekman, R. Stoffer and G. Jobst, Opt Express, 2014, 22, 8856-8870.

36. K. Misiakos, I. Raptis, E. Makarona, A. Botsialas, A. Salapatas, P. Oikonomou, A. Psarouli, P. S. Petrou, S. E. Kakabakos, K. Tukkiniemi, M. Sopanen and G. Jobst, Opt Express, 2014, 22, 26803-26813.

37. Q. Liu, X. Tu, K. W. Kim, J. S. Kee, Y. Shin, K. Han, Y.-J. Yoon, G.-Q. Lo and M. K. Park, Sensors and Actuators B: Chemical, 2013, 188, 681-688.

38. X. Tu, J. Song, T.-Y. Liow, M. K. Park, J. Q. Yiying, J. S. Kee, M. Yu and G.-Q. Lo, Optics Express, 2012, 20, 2640-2648.

39. T. Chalyan, R. Guider, L. Pasquardini, M. Zanetti, F. Falke, E. Schreuder, R. Heideman, C. Pederzolli and L. Pavesi, Biosensors, 2016, 6, 1.

40. The Pocket Project, http://www.pocket-proj.eu//.

41. V. Dugas, A. Elaissari and Y. Chevalier, in Recognition Receptors in Biosensors, ed. M. Zourob, Springer New York, 2010, DOI: 10.1007/978-1-4419-0919-0_2, ch. 2, pp. 47134.

42. H. Vaisocherova, E. Brynda and J. Homola, Anal Bioanal Chem, 2015, 407, 3927-3953.

43. M. Zourob, Recognition receptors in biosensors, Springer, New York ; London, 2010.

44. B. H. Schneider, J. G. Edwards and N. F. Hartman, Clin Chem, 1997, 43, 1757-1763.

45. K. E. Zinoviev, A. B. Gonzalez-Guerrero, C. Dominguez and L. M. Lechuga, J Lightwave Technol, 2011, 29, 1926-1930. 
46. J. García-Rupérez, V. Toccafondo, M. J. Bañuls, J. G Castelló, A. Griol, S. Peransi-Llopis and Á. Maquieira, Opt. Express, 2010, 18, 24276-24286.

47. E. Mauriz, M. C. García-Fernández and L. M. Lechuga, TrAC Trends in Analytical Chemistry, 2016, 79, 191-198.

48. M. J. Banuls, R. Puchades and A. Maquieira, Analytica Chimica Acta, 2013, 777, 1-16.

49. G. T. Hermanson, in Bioconjugate Techniques (Third edition), ed. G. T. Hermanson, Academic Press, Boston, 2013, DOI: http://dx.doi.org/10.1016/B978-0-12-3822390.00003-0, pp. 229-258.

50. T. Kumeria, A. Santos and D. Losic, Acs Appl Mater Inter, 2013, 5, 11783-11790.

51. Y. R. Paz, Beilstein J Nanotech, 2011, 2, 845-861.

52. M. Cypryk and Y. Apeloig, Organometallics, 2002, 21, 21652175.

53. L. Wu, L. Cai, A. Liu, W. Wang, Y. Yuan and Z. Li, Applied Surface Science, 2015, 349, 683-694.

54. O. Scheler, J. T. Kindt, A. J. Qavi, L. Kaplinski, B. Glynn, T. Barry, A. Kurg and R. C. Bailey, Biosensors and Bioelectronics, 2012, 36, 56-61.

55. H. W. Choi, H. Takahashi, T. Ooya and T. Takeuchi, Analytical Methods, 2011, 3, 1366-1370.

56. K. Ma, T. Ekblad, M. Koerkamp, H. Kelderman, M. van Wijlen, A. Duarah, J. Yue, L. Zhang, M. Wong and M. Lim, Int J Environ an Ch, 2015, 95.

57. A. Densmore, M. Vachon, D. X. Xu, S. Janz, R. Ma, Y. H. Li, G. Lopinski, A. Delâge, J. Lapointe, C. C. Luebbert, Q. Y. Liu, P. Cheben and J. H. Schmid, Opt. Lett., 2009, 34, 35983600.

58. K. C. Popat, S. Sharma, R. W. Johnson and T. A. Desai, Surface and Interface Analysis, 2003, 35, 205-215.

59. H. K. Hunt, C. Soteropulos and A. M. Armani, Sensors-Basel, 2010, 10, 9317-9336.

60. A. Ksendzov and Y. Lin, Opt. Lett., 2005, 30, 3344-3346.

61. S. Lee, S. C. Eom, J. S. Chang, C. Huh, G. Y. Sung and J. H. Shin, Opt. Express, 2010, 18, 20638-20644.

62. Y. B. Guo, J. Y. Ye, C. Divin, B. H. Huang, T. P. Thomas, J. R. Baker and T. B. Norris, Analytical Chemistry, 2010, 82, 5211-5218.

63. N. Massad-Ivanir, G. Shtenberg, A. Tzur, M. A. Krepker and E. Segal, Analytical Chemistry, 2011, 83, 3282-3289.

64. A. B. Gonzalez-Guerrero, M. Alvarez, A. G. Castano, C. Dominguez and L. M. Lechuga, Journal of Colloid and Interface Science, 2013, 393, 402-410.

$65 . \quad$ B. H. Schneider, E. L. Dickinson, M. D. Vach, J. V. Hoijer and L. V. Howard, Biosensors and Bioelectronics, 2000, 15, 1322.

66. K. De Vos, J. Girones, S. Popelka, E. Schacht, R. Baets and P. Bienstman, Biosensors and Bioelectronics, 2009, 24, 25282533.

67. J. Escorihuela, M. Bañuls, J. Castelló, V. Toccafondo, J. García-Rupérez, R. Puchades and Á. Maquieira, Anal Bioanal Chem, 2012, 404, 2831-2840.

68. B. Sepúlveda, J. S. d. Río, M. Moreno, F. J. Blanco, K. Mayora, C. Domínguez and L. M. Lechuga, Journal of Optics A: Pure and Applied Optics, 2006, 8, S561.

69. J. A. Howarter and J. P. Youngblood, Langmuir, 2006, 22 $11142-11147$.

70. P. Thissen, O. Seitz and Y. J. Chabal, Progress in Surface Science, 2012, 87, 272-290.
71.

P. K. Sarswat, A. Sathyapalan and M. L. Free, in AntiAbrasive Nanocoatings, ed. M. Aliofkhazraei, Woodhead Publishing, 2015, DOI: http://dx.doi.org/10.1016/B978-085709-211-3.00013-3, pp. 331-348.

72. A. Arafat, M. Giesbers, M. Rosso, E. J. R. Sudhölter, K. Schroën, R. G. White, L. Yang, M. R. Linford and H. Zuilhof, Langmuir, 2007, 23, 6233-6244.

73. A. Arafat, K. Schroën, L. C. P. M. de Smet, E. J. R. Sudhölter and H. Zuilhof, Journal of the American Chemical Society, 2004, 126, 8600-8601.

74. L. Scheres, A. Arafat and H. Zuilhof, Langmuir, 2007, 23, 8343-8346.

75. T. Böcking, K. A. Kilian, K. Gaus and J. J. Gooding, Advanced Functional Materials, 2008, 18, 3827-3833.

76. H. Qiao, B. Guan, J. J. Gooding and P. J. Reece, Opt. Express, 2010, 18, 15174-15182.

77.

L. De Stefano, P. Arcari, A. Lamberti, C. Sanges, L. Rotiroti, I. Rea and I. Rendina, Sensors-Basel, 2007, 7, 214-221.

78. M.-J. Bañuls, V. González-Pedro, C. A. Barrios, R. Puchades and Á. Maquieira, Biosensors and Bioelectronics, 2010, 25, 1460-1466.

79. C. A. Barrios, M. J. Bañuls, V. González-Pedro, K. B. Gylfason, B. Sánchez, A. Griol, A. Maquieira, H. Sohlström, M. Holgado and R. Casquel, Opt. Lett., 2008, 33, 708-710.

80. C. F. Carlborg, K. B. Gylfason, A. Kazmierczak, F. Dortu, M. J. Banuls Polo, A. Maquieira Catala, G. M. Kresbach, H. Sohlstrom, T. Moh, L. Vivien, J. Popplewell, G. Ronan, C. A. Barrios, G. Stemme and W. van der Wijngaart, Lab on a Chip, 2010, 10, 281-290.

81. R. P. Gandhiraman, V. Gubala, L. C. H. Nam, C. Volcke, C. Doyle, B. James, S. Daniels and D. E. Williams, Colloids and Surfaces B: Biointerfaces, 2010, 79, 270-275.

82. J. Xu, D. Suarez and D. Gottfried, Anal Bioanal Chem, 2007, 389, 1193-1199.

83. E. Brynda, M. Houska, A. Brandenburg and A. Wikerstal, Biosens Bioelectron, 2002, 17, 665-675.

84. B. H. Schneider, E. L. Dickinson, M. D. Vach, J. V. Hoijer and L. V. Howard, Biosensors and Bioelectronics, 2000, 15, 597604.

85. T. Špringer, M. Bocková and J. Homola, Analytical Chemistry, 2013, 85, 5637-5640.

86. S. Lowe, N. M. O'Brien-Simpson and L. A. Connal, Polymer Chemistry, 2015, 6, 198-212.

87. C. Nogues, H. Leh, J. Lautru, O. Delelis and M. Buckle, PLoS ONE, 2012, 7, e44287.

88. M. Soler, M.-C. Estevez, M. Alvarez, M. Otte, B. Sepulveda and L. Lechuga, Sensors-Basel, 2014, 14, 2239.

89. O. Pop-Georgievski, Š. Popelka, M. Houska, D. Chvostová, V. Proks and F. Rypáček, Biomacromolecules, 2011, 12, 3232-3242.

90. S. Chen, J. Zheng, L. Li and S. Jiang, Journal of the American Chemical Society, 2005, 127, 14473-14478.

91. Z. Zhang, S. Chen and S. Jiang, Biomacromolecules, 2006, 7, 3311-3315.

92. C. Gao, G. Li, H. Xue, W. Yang, F. Zhang and S. Jiang, Biomaterials, 2010, 31, 1486-1492.

93. N. D. Brault, C. Gao, H. Xue, M. Piliarik, J. Homola, S. Jiang and Q. Yu, Biosensors and Bioelectronics, 2010, 25, 22762282.

94. A. B. González-Guerrero, Thesis: Bimodal waveguide interferometer device based on silicon photonics 


\section{ARTICLE}

2

technology for label-free and high sensitive biosensing by the Autonomous University of Barcelona, 2012.

95. T. Jartti, M. Söderlund-Venermo, K. Hedman, O. Ruuskanen and M. J. Mäkelä, Paediatric Respiratory Reviews, 2013, 14, 38-45.

96. A. Ymeti, J. Greve, P. V. Lambeck, T. Wink, H. van, Beumer, R. R. Wijn, R. G. Heideman, V. Subramaniam and J. S. Kanger, Nano Letters, 2007, 7, 394-397.

97. A. A. Kondkar and K. K. Abu-Amero, BioMed Research International, 2015, 2015, 10.

98. S. Shrivastava, A. Mukherjee and R. B. Ray, World Journal of Hepatology, 2013, 5, 479-486.

99. M. Pichler and G. A. Calin, Br J Cancer, 2015, 113, 569-573.

100. C. S. Huertas, D. Fariña and L. M. Lechuga, ACS Sensors, 2016, 1, 748-756.

101. P. L. Edmiston, D. P. Campbell, D. S. Gottfried, J. Baughman and M. M. Timmers, Sensors and Actuators B: Chemical, 2010, 143, 574-582.

102. K. Ma, T. Ekblad, M. K. Koerkamp, H. Kelderman, M. v. Wijlen, A. Duarah, J. Yue, L. Zhang, M. V.-M. Wong and M. H. Lim, Int J Environ an Ch, 2015, 95, 366-378.

103. Y. Wang, Y. Zheng, F. Yang and X. Yang, Chemical Communications, 2012, 48, 2873-2875.

104. World Health Organization. Chapter 8: Chemical aspects in Guidelines for drinking-water quality, http://www.who.int/water sanitation health/dwq/gdwq 3rev/en/l).

105. BRAAVOO Project, http://www.braavoo.org/).

106. S. Yang, A. Undar and J. D. Zahn, Lab Chip, 2006, 6, 871880.

107. I. K. Dimov, L. Basabe-Desmonts, J. L. Garcia-Cordero, B. M. Ross, A. J. Ricco and L. P. Lee, Lab on a Chip, 2011, 11, 845850.

108. K. Tae Goo, Y. Yong-Jin, J. Hongmiao, L. Pei Yi and C. Yu, Journal of Micromechanics and Microengineering, 2014, 24, 087001.

109. T. C. Marentis, B. Kusler, G. G. Yaralioglu, S. Liu, E. O. Haeggström and B. T. Khuri-Yakub, Ultrasound Med Biol, 2005, 31, 1265-1277.

110. Q. Tseng, A. M. Lomonosov, E. E. Furlong and C. A. Merten, Lab Chip, 2012, 12, 4677-4682. 